Acta Cryst. (2002). A58 (Supplement), C191

THE GTPase SWITCH: A FAMILIAR, CONSERVED MODULE WITH UNEXPECTED VARIATIONS

A. Wittinghofer

Max-Planck-Institut Fuer Molekulare Physiologie Otto-Hahn-Strasse 11 DORTMUND 44227 GERMANY

GTP-binding proteins cycle between a GDP-bound inactive state and a GTPbound active state. The switch-ON reaction involves the exchange of tightly bound GDP against GTP, while the switch-OFF mechanism involves the enzymatic cleavage of GTP to GDP. Usually the first reaction is catalyzed by guanine nucleotide exchange factors GEFs, while the second is activated by GTPase-activating proteins GAPs. Much work has been devoted to analyzing the details of the GTPase reaction, which appeared to be using a conserved mechanism. Part of the motivation came from the finding that the inability of certain of these proteins to hydrolyze GTP has drastic consequences for the cell. For the small $\mathrm{G}$ proteins Ras and Rho and for the G-alpha subunits of heterotrimeric $G$ proteins it was shown conclusively that the mechanism of GTP hydrolysis employs a general mechanism whereby a glutamine and an arginine residue are the two most important residues for catalysis. In the case of Ras and Rho the arginine is supplied in trans by the respective RasGAP and RhoGAP. Recent studies with a number of GTP-binding proteins suggest that there are a variety of mechanisms used for switching between the GTP- and GDP-bound states, and that different mechanisms for the chemical step of the reaction have to be considered. A few examples will be presented.
Acta Cryst. (2002). A58 (Supplement), C191

\section{CHEMICAL BONDING AS ELECTRONIC COHERENCE}

\section{W. Weyrich}

Department of Chemistry, University of Konstanz, Germany

The access to the microscopic structure of samples is provided in principle by probing them with photons or other particles and analyzing their scattering intensity distribution as a function of energy and momentum transfer (Bethe surface). Diffraction work extracts particles with zero energy transfer and converts the momentum-transfer information into a time-averaged static structure (spatial electron, spin or magnetization density distributions). Maxima between covalently bonded atoms are the most prominent features in difference densities formed by subtraction of atomic contributions. Their origin is the interference between phase-locked atomic wave functions or, in other words, atomic quantum-mechanical coherence. A powerful tool for the quantitative and much more detailed and sensitive description of coherence is the offdiagonal part of density matrices, as demonstrated with examples. Coherence is not restricted to covalent bonds, but reflects the entanglement of constituents and thus chemical bonding in its most general sense. The off-diagonal part of density matrices is experimentally visible in the momentum density, i.e. in the electronic motion, mostly determined by high-energy-transfer (= Compton) scattering. The sensitivity to analyze even hydrogen bonds has led to the idea to reconstruct density matrices from combined experimental position and momentum densities. That combination of information from different experiments gives access to the full single-particle description of the electronic structure of a substance, which consists of the electron density and its internal quantum-mechanical coherence.

\section{Keywords: CHEMICAL BONDING, COHERENCE, DENSITY MATRICES}

Acta Cryst. (2002). A58 (Supplement), C191

\section{STEREOCHEMICAL APRROACH TO CRYSTAL NUCLEATION}

M. Lahav ${ }^{1}$ L. Leiserowitz ${ }^{2}$

Weizmann Institute of Science Materials and Interfaces. Weizamnn Institute of Science Rehovot 76100 ISRAEL

Crystallization processes investigated during the years primarily via kinetic and thermodynamic methods did not always take into proper account the structures of the crystals and their surfaces. Surfaces that delineate growing and dissolving crystals might be considered as being composed of structured 'active-sites' that interact with molecules present in solution. Auxiliary molecules designed to interact stereo-specifically and enantio-selectively with these active sites, act as efficient inhibitors for crystal growth and dissolution. According to classical nucleation theory, when a solution enters the nonequilibrium supersaturated regions, the molecules of the solute form first aggregates that are consequently converted into crystalline nuclei, which cross a critical size to convert into 3-d crystals. A working hypothesis suggesting that these nuclei assume, at the onset of crystallization, a structure akin to these of the 3-D mature crystals. for that reason, molecules that are recognized by the surfaces of the crystals should be also recognized by the surfaces of the embryonic nuclei. Consequently, in the presence of the inhibitors the nuclei will undergo decomposition instead of crossing the critical size en route to crystal formation. Examples will include control of crystal polymorphism, and design of a process for large-scale resolution of amino acids by removal of lamella twinning. An insight of the early stages of crystallization was provided by studying structure and dynamics of 2-d crystallites of amphiphilic molecules that serve as stereo-specific crystal nucleation promoters for crystallization. The critical size of the nuclei of hexagonal ice grown in the presence of amphiphilic alcohols was estimated.
Acta Cryst. (2002). A58 (Supplement), C191

\section{THE CONTRIBUTION OF DIRECT METHODS TO} MACROMOLECULAR STRUCTURE DETERMINATION

\section{G.M. Sheldrick}

Universitaet Goettingen Lehrstuhl Fuer Strukturchemie Tammannstrasse 4 Goettingen D-37077 GERMANY

The large majority of small molecule structures are solved by so-called direct methods, often in less than a minute of computer time. Since the introduction of the dual-space approach by the Buffalo group in 1993 and alternatives introduced later by de Graff and Giacovazzo, direct methods are capable of solving structures containing up to about 1000 unique atoms, and even larger structures can be solved if heavier atoms are present. From the point of view of macromolecular crystallography, the main disadvantage of these methods is that they require diffraction data to 'atomic resolution', i.e. about $1.2 \AA$, and a relatively small fraction of macromolecular crystals diffract so well. However the same methods can be used to solve heavy-atom substructures from MAD, SIR or SAD data at much lower resolution (3-5 $\AA$ ): since the sites are in general further apart than this and the data to parameter ratio is much higher, the resolution restriction does not apply. The success rate can be improved by about an order of magnitude by incorporating information from the Patterson. This will be illustrated by the programs SHELXD (for both $a b$ initio phasing and substructure solution) and SHELXE (phase determination) that in favorable cases can create interpretable maps within a few minutes starting from single- or multi-wavelength diffraction data of macromolecules containing anomalous scatterers.

Keywords: DIRECT METHODS MAD PHASING SAD PHASING

Keywords: CRYSTAL NUCLEATION, STEREOCHEMISTRY, 2-D CLUSTERS. 